\title{
Advanced Axial Pumps for Oil Industry
}

\author{
Yakov Mikhajlovich Kashin \\ Department of Electrical Engineering and Electrical \\ Machines \\ Kuban State Technological University \\ Krasnodar, Russia \\ jlms@mail.ru
}

\author{
Alexander Valeryevich Samorodov \\ Department of Electrical Engineering and Electrical \\ Machines \\ Kuban State Technological University \\ Krasnodar, Russia \\ Alex.Samorodoff@gmail.com
}

\author{
Bogaudin Khamidovich Gaitov \\ Department of Electrical Engineering and Electrical \\ Machines \\ Kuban State Technological University \\ Krasnodar, Russia \\ kkllev1@mail.ru
}

\author{
Vladislav Anatolievich Kim \\ Department of Electrical Engineering and Electrical \\ Machines \\ Kuban State Technological University \\ Krasnodar, Russia \\ vladk-kub@mail.ru
}

\author{
Mikhail Sergeyevich Khristoforov \\ Department of Electrical Engineering and Electrical Machines \\ Kuban State Technological University \\ Krasnodar, Russia \\ khristoforov87@mail.ru
}

\begin{abstract}
The authors conducted a review of a number of pumps for pumping oil and oil products of a traditional cylindrical (radial) construction. Their critical analysis is presented in the paper. In addition, the shortcomings and advantages of the pump are described. The designs of axial centrifugal pumps were developed by the authors for pumping petroleum products, allowing one to protect the stator winding of the pump motor against harmful mechanical and chemical influences of the pumped liquid, to reduce the gap between the stator and the rotor of the pump motor by increasing the rigidity of the structure, to improve the weight and size indicators of the pump unit, to reduce its cost, to simplify the design and to increase its reliability in general, and also to reduce energy losses and, accordingly, to increase the efficiency with decreasing heating of the pumped liquid.
\end{abstract}

Keywords - axial centrifugal motor-pump, pumped oil products.

\section{INTRODUCTION}

Pumps serving for the transfer of liquid petroleum products belong to a special class of industrial pumps [1-10], the use of which is an indispensable component of the oilproducing and oil-refining industry.

It is known that a pump [1], designed for the transfer of petroleum products (oil, fuel oil, diesel fuel, kerosene), does not contain mechanical impurities. Such pump includes (Fig.1) the front and rear covers, the body with the wells, in which the driving and driven gears are installed. They are integral with the shafts and rotate in the bearings, installed in these wells. Expansion joints with 3-shaped cuffs compensate for the end clearances between gears and bearings, the internal cavity of which is connected to the discharge pressure. There is also the mechanical seal.

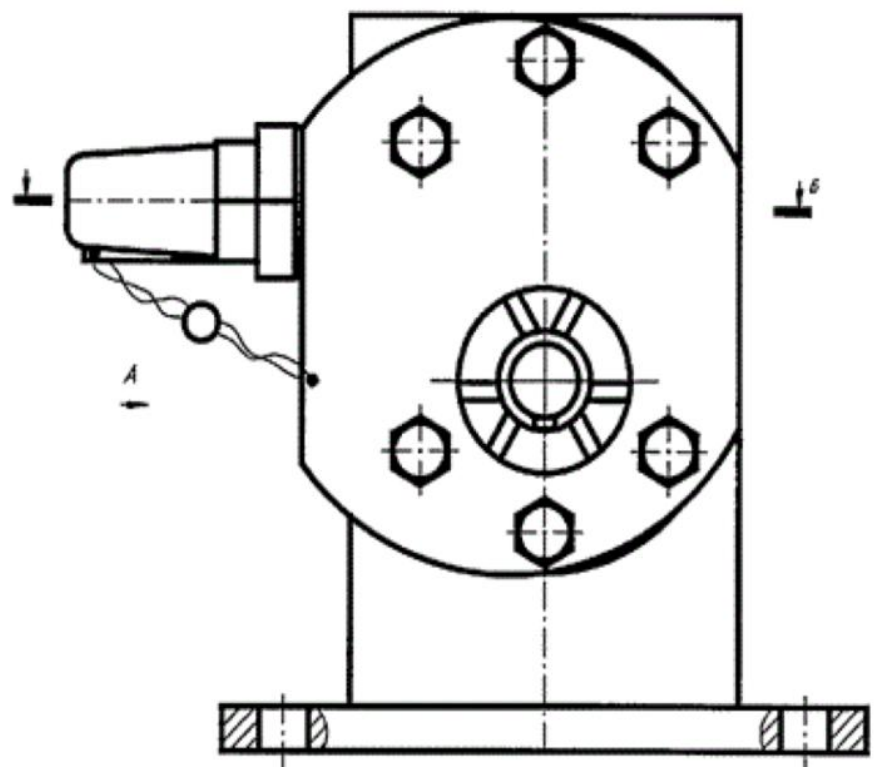

Fig. 1. Scheme of pump for transfer of liquid petroleum products

The pump (Figure 1) is equipped with a safety valve for the transfer of working fluid from the high-pressure zone to the suction zone when the pressure in the discharge pipeline exceeds the permissible level. The mechanical seal consists of a thrust bearing, fixedly mounted in the front cover and sealed 
on the outer surface by a rubber ring. There is also a heel, sealed on the shaft of the driving gear by a rubber ring and pressed into the thrust spring by a spring, installed in the axial hole of the shaft of the drive gear that rests on one side in the stationary pin. It is mounted on the shaft of the drive gear, and on the other hand, into the movable pin, connected to the bearing plate and placed in a diametral hole on the shaft of the drive gear. The back cover is provided with a bracket for fastening the pump.

There is also another pump known [3], which can also be used for pumping petroleum products. It comprises housing 1, in which a rotor is mounted in the form of a shaft 2 with an impeller 3 and a guide apparatus 4 . The housing 1 is provided with a spiral branch including two turns 5 and 6, external and internal, respectively. Inner coil 6 has interconnected input scroll portion 7 and output diffuser portion 8 in the turn. Outer coil 5, input spiral portion 9, and output diffuser portion 10 are connected to each other via bypass portion 11 . The initial cross-section of outer coil 5 is formed firstly along the twist spiral, and inner coil 6 is located behind the first one with a spiral offset and is combined in the radial-axial plane of the rotor with the initial section of bypass section 11 of outer coil 5. The sum of their areas equal to the cross-sectional area of the outlet section of scroll portion 9 of outer coil 5 , and the sum of similar cross-sectional areas of primary coil 5 and outer adjacent thereto diffuser portion 8 of inner coil 6 equals the area of the output cross-section of spiral portion 7 of inner coil 6.

Outer and inner windings 5 and 6 are separated by inner wall 12. Inner drain wall 12 in the separation zone of bypass section 11 of outer turn 5 and spiral section 7 of inner turn 6 is spiral-cylindrical.

The shape of the body of such pump with a two-turn twisting of the pumped liquid flow with the configuration of the spiral, bypass and diffuser sections and the ratio of the areas of their initial and outlet cross sections makes it possible to increase the reliability and lifespan of the pump.

However, the pumps and other pumps of the radial design discussed above have a number of drawbacks inherent in all radial electric machines.

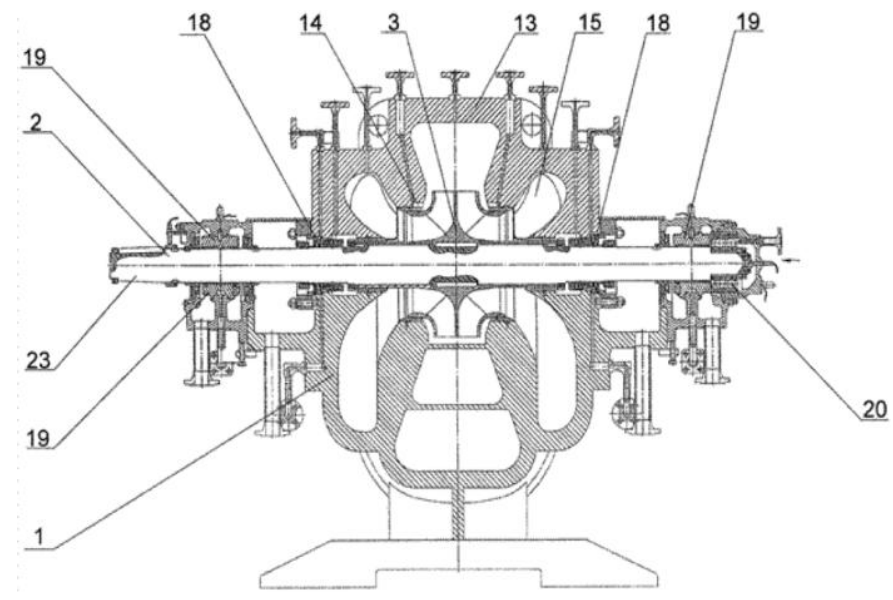

Fig. 2. Cylindrical motor-pump
Recently, a class of electric machines of axial construction for various purposes, including pumps, has been actively developing. This is due to a number of advantages of such machines in comparison with machines of radial construction: smaller sizes; greater rigidity of the structure; favorable conditions of heat transfer, cooling and ventilation; a significant simplification of the winding work due to the open tooth zone; practically no-waste use of magnetic materials; convenience of articulation with the mechanism and drive; comparative ease of operation and repair, etc. Such electric machines include motor pumps, too [8-10].

In this regard, it is very important to develop efficient centrifugal pumps of axial design.

\section{RESULTS AND DISCUSSION}

A motor-pump, developed under the guidance of prof. Gaitov B.Kh., comprising an electric motor stator, which is a magnetic core with grooves in which the primary winding is laid, is known. There is also a massive rotor-impeller, representing a hollow drum where inner surface of the axle pump shafts are arranged.

The structural and technological features of such pump, as well as other cylindrical pumps, assume a somewhat longer construction, which in practice leads to a longer presence of the pumped liquid in the massive rotor-impeller. This impeller, due to its electromagnetic characteristics, heats the pumped liquid. The latter is not always acceptable, because it can lead to a change in some properties of pumped oil and petroleum products.

Reducing the same length of the cylindrical motor-pump (MP) (without increasing its diameter) leads to a decrease in the power of the pumping unit.

The design elongation of the cylindrical (radial) MP often also creates difficulties in their placement and arrangement together with other equipment. Thus, it may be necessary to have a small machine length with an increased diameter, which, as noted above, can not be performed with a cylindrical (radial) motor-pump design.

Analysis of the above-stated disadvantages allows us to come to a simple, at first glance, solution, namely: replace the cylindrical structure of MP by an axial one with a single stator, as shown in Fig. 3.

However, the performance of a single-stator motor-pump in the operating mode under full load gives good results. When carrying out the same maintenance work, when it is necessary to ensure only rotation of the rotor-impeller without load (without feeding the pumped liquid), at reduced speeds (up to 1-2 rpm) and pump operation under partial load, it is not necessary to use fully the stator power. This would lead to unjustified energy consumption and shorten the life of the device.

A superficial view of this problem seems to suggest a classic way out, namely: the use of pole-switched windings. However, such windings allow one to reduce the speed and power consumption of the engine in a ratio of 2:1 (providing other ratios dramatically complicates the design and requires 
special equipment, which makes it impossible to obtain these ratios).

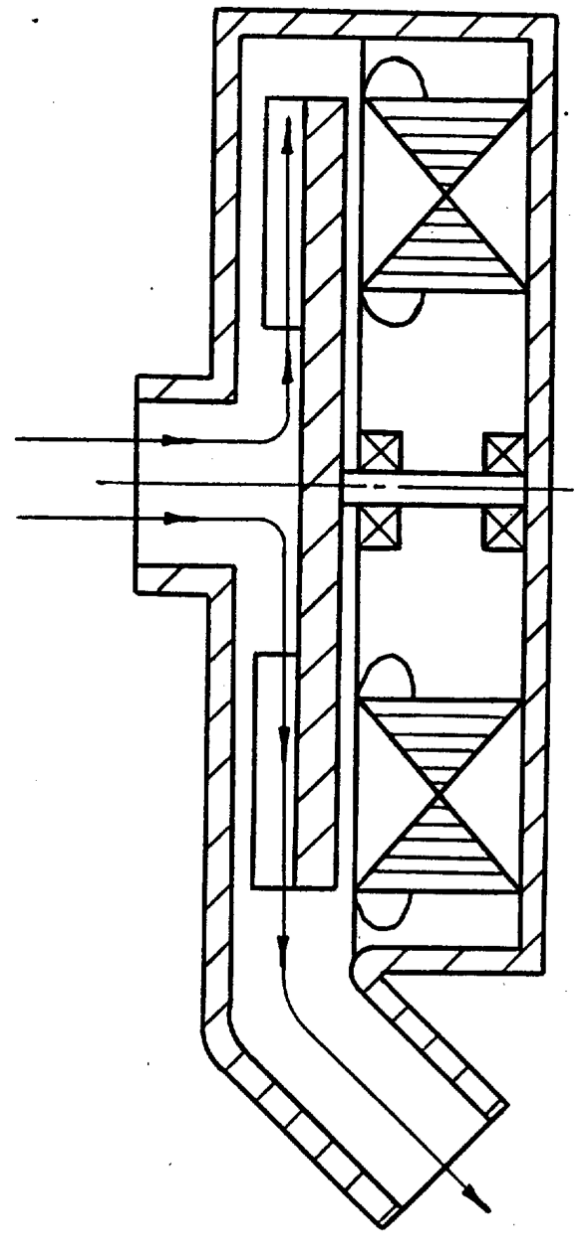

Fig. 3. Pump's cross-section

In addition, replacing a single-stator cylindrical motor pump with a single-shaft axial motor-pump cannot always give a positive effect on ensuring the discreteness of D-N power supply and increasing the power parameters of the installation. It should be noted that the energy parameters ( $\cos \varphi$ and efficiency) of the motor-pump essentially depend on the air gap size, i.e. the size of the air gap should be optimal for a given size of the machine.

For each operating mode, there is an optimum air gap value. When starting (increasing the load on the shaft), the air gap is larger than at the rated load.

The magnitude of the air gap in the axial MP structure can be self-adjusted and influenced by the operating mode of the machine, i.e. when the load on the shaft increases (increasing the flow of the pumped liquid or increasing its viscosity), the stator current increases, which in turn leads to an increase in the magnetic interaction between the stator and the rotorimpeller. The latter can lead to a sharp decrease in the air gap, down to zero, which is certainly unacceptable. It should be noted that at start-up, a large amount of air gap leads to a decrease in the starting current, while reducing the installed power of the electric motor; and in the operating mode will reduce the energy performance. The small value of the air gap causes, at start-up, an increase in the starting current, and in the operating mode, to an increase in energy indicators.

Improvement of overall dimensions and a slight increase in MP energy indicators is possible using a two-stator pump design in which the rotor is made in the form of interconnected two rotor disks located between the end faces of two stators of the electric motor [8]

This MP design ensures the stability of the machine air gap, but does not ensure the adjustment of the air gap and the optimality of energy performance.

The pump for the petroleum products transfer does not have the above mentioned drawbacks. It comprises a body, motor stators mounted therein and a pump impeller that is a rotor of the electric motor and made in the form of interconnected two rotor disks disposed between two end surfaces of two motor stators with the necessary air gaps, one of the stators of the electric motor is disconnected from the power supply network independently of the other, and the pump is equipped with a shaft fixed in the bearing body on which it is fixed the impeller.

Fig. 4 shows such engine-pump. The motor-pump shown in Fig. 2, consists of a housing 1 with inlet 2 and outlet branch pipe 3 . On the body 1 two end-type stators 4 with windings 5 are fixed. On the shaft 6 which is fixed in the bearing assemblies 7 , there are two rotor-disks 8 , which are constitute the impeller of the pump and fastened between the blades 9 . Between the stators 4 and the rotor disks 8 , there are working air gaps 10 .

The thickness of the rotor-disks in such an engine-pump is greater than the depth of electromagnetic wave penetration. This design feature ensures that the blades-rods do not strike the field pattern in the rotor, remaining, however, an additional and highly developed cooling surface of the rotors, ensuring equalization of the thermal potential between the two rotor-disks.

The arrows indicate the motion of the pumped liquid. The pump shown in Fig. 4 operates as follows. The winding 5 of stators 4 is supplied with a three-phase voltage, which creates rotating magnetic fields. Moreover, the windings 5 of the stators 4 are connected so that the magnetic fields rotate in the opposite direction, which, in the mirror arrangement of the stators, actually ensures their rotation in one direction. Rotating fields in the stators induce eddy currents (in their "own") rotor-currents in the rotors-discs 8 . The interaction of the eddy currents of the rotor 8 with the rotating magnetic fields of the "own" stators 4 creates rotating moments which are transmitted to the common shaft 6 fixed in the bearing assemblies 7 .

Rotation of the rotor-discs 8 with blades 9 ensures the pumping of liquid from the inlet nozzles 2 to the outlet 3 .

It should be noted that when a three-phase voltage is applied to the windings 5 of the stators 4 and when the rotating magnetic field is created, not only the rotational moment is created, but also the forces of magnetic tension 
between the stator and the "own" rotor are created. And since the two stators 4 are performed with the same electromagnetic parameters and the rotor disks are also performed in the same way, the magnetic pulling forces created by the two stators will balance each other, not allowing one to decrease (increase) the values of the two air gaps 10 .

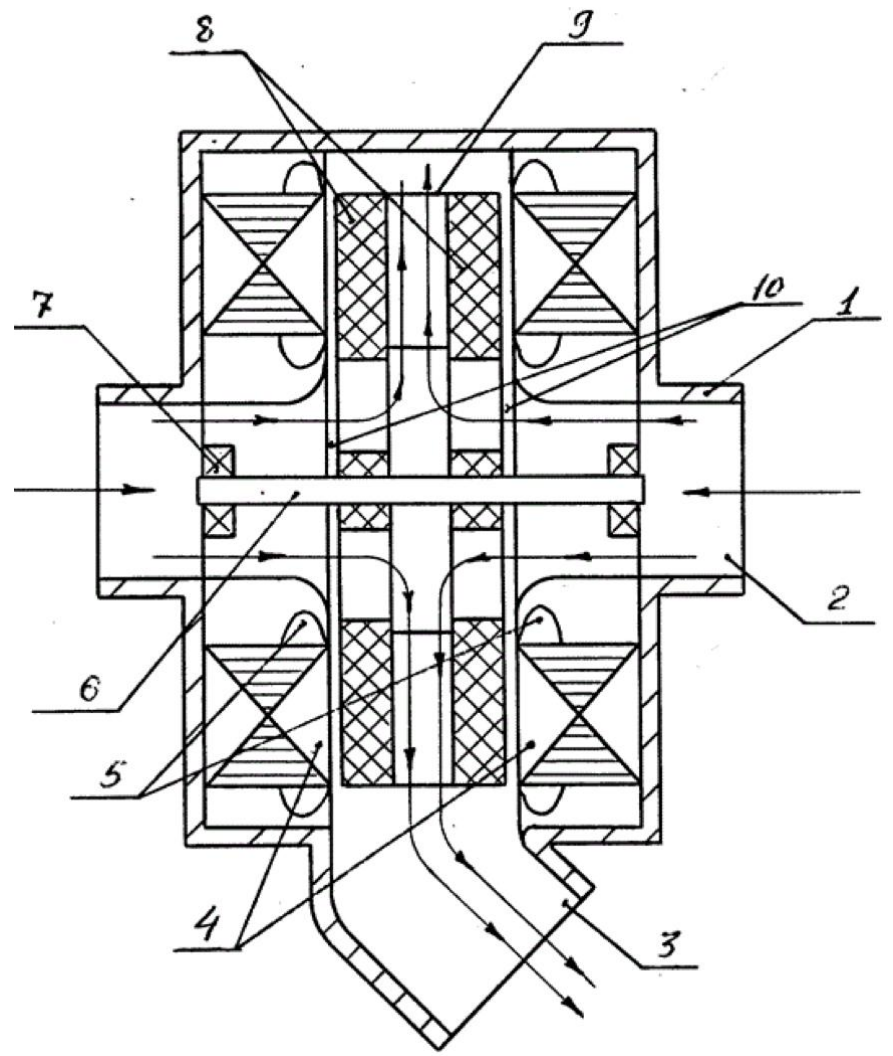

Fig. 4. Motions of pumped liquid

That is, when the pump starts or when the viscosity of the pumped liquid changes, when the moment of resistance is high and the machine needs an increased torque, two stators operate, providing the necessary torque on the shaft. Simultaneous operation of the two stators does not allow changing the air gap, which would lead to an increase in magnetic losses and, consequently, to a decrease in energy indicators.

After the pump is turned on, its output goes to the working frequency of rotation and with a decrease in the dynamic moment $[\mathrm{Mg} \mathbf{J}(\mathrm{dw} / \mathrm{dt})$, where $\mathbf{J}$ is the moment of mechanism inertia, $w$ is the angular frequency of rotation of the mechanism, $t$ is the time], one stator turns off, which causes, due to the forces of magnetic traction, reduction of the air gap between the operating stator and its "rotor-disk", losses are reduced and energy indicators are improved.

Therefore, when the machine is operating in the nominal (operating) mode, when it is not necessary to use two stators. Switching off one stator from the network decreases the power consumption due to a reduction in the air gap between the running stator and the rotor. This leads to a reduction in losses, which ultimately results in increased efficiency and $\cos \varphi$.
However, the design of such pump does not provide protection of the stator winding from harmful mechanical and chemical influences of the pumped liquid, which can lead to damage to the insulation of the stator winding with subsequent shorting of the winding. A significant drawback of such a pump is the presence of massive rotor discs made of structural steel, resulting in unjustifiably large power losses in the eddy currents in the array of rotor disks and magnetic hysteresis, which leads to strong heating of the rotor and, therefore, to large energy losses and significant reduce of the pump efficiency. The absence of windings on the rotor-discs reduces the energy indicators of the pump as a whole. In addition, steel massive rotor-disks have a large mass, as a result of which the mass-size parameters of the pump unit as a whole deteriorate, the role of windings is performed by structural steel having a large active resistance. The shaft connecting the rotors of such pump must have a sufficiently large diameter and, correspondingly, a large mass for the transmission of twisting and bending moments, axial and radial forces, which also leads to a deterioration in the mass-dimensions of the pump.

To protect the stator winding of the pump motor from the harmful mechanical and chemical influences of the pumped liquid, to reduce the heating of the pumped liquid and the pump as a whole, to improve energy performance, to reduce energy losses, to improve efficiency and to improve weight and size indicators of the pump set, an axial centrifugal motorpump has been developed [9].

The general view of the axial centrifugal motor-pump in the section is shown in Fig. 3.

Stator 2 of the motor-pump motor, mounted inbody 1 , is hermetically separated from flow-through part 5 of the pump motor by thin membrane 6 made of a dielectric material and protecting three-phase stator winding 3 from the harmful mechanical and chemical effects of the pumped liquid. Axial magnetic circuit 8 of the rotor is made of electric steel with short-circuited aluminum winding 7 and is pressed into an impeller made of light aluminum alloy 4 of a motor-pump with blades. A freely rotating impeller-rotor, obtained in such way and belonging to the motor-pump, is mounted freely rotating on axis 9 , fixed in the body.

The axial centrifugal motor-pump works as follows. When three-phase winding 3 of stator 2 of the electric motor is connected to the three-phase supply network, a rotating electromagnetic moment is created according to the wellknown principle of operation of the induction motor, which drives impeller-rotor 4 into rotation. When impeller-rotor 4 rotates, the liquid in flow-through part 5, entrained by impeller-rotor 4 , also comes into rotation. At the same time, the liquid in flow-through part 5 is acted upon by centrifugal forces that pump fluids according to the well-known principle of operation of conventional centrifugal pumps.

The developed design, in contrast to the known ones, allows one to protect the stator winding of the axial centrifugal motor-pump from the harmful effect of the pumped liquid by means of a membrane of dielectric material. In addition, it results in the reduction of heating the pumped liquid by reducing the eddy current and magnetic hysteresis losses, an increase of the overall pump power performance of the rotor 
working wheel with the magnetic core containing winding. It also enables a reduction of energy losses and an increase of the efficiency due to reduced losses of magnetic hysteresis. Magnetic hysteresis losses are reduced in the proposed design due to the magnetic properties of the electrical steel from which the rotor magnetic circuit is made. To reduce the power loss by eddy currents makes it possible to perform the magnetic circuit of the impeller-rotor with a sham. Improvement of the overall dimensions of the pump is ensured by the partial performance of the rotor impeller made of light aluminum alloy and by the transmission of the torque due to electromagnetic forces, which makes it possible to replace the drive shaft having a large diameter and weight, respectively, to a fixed small diameter axis and small masses.

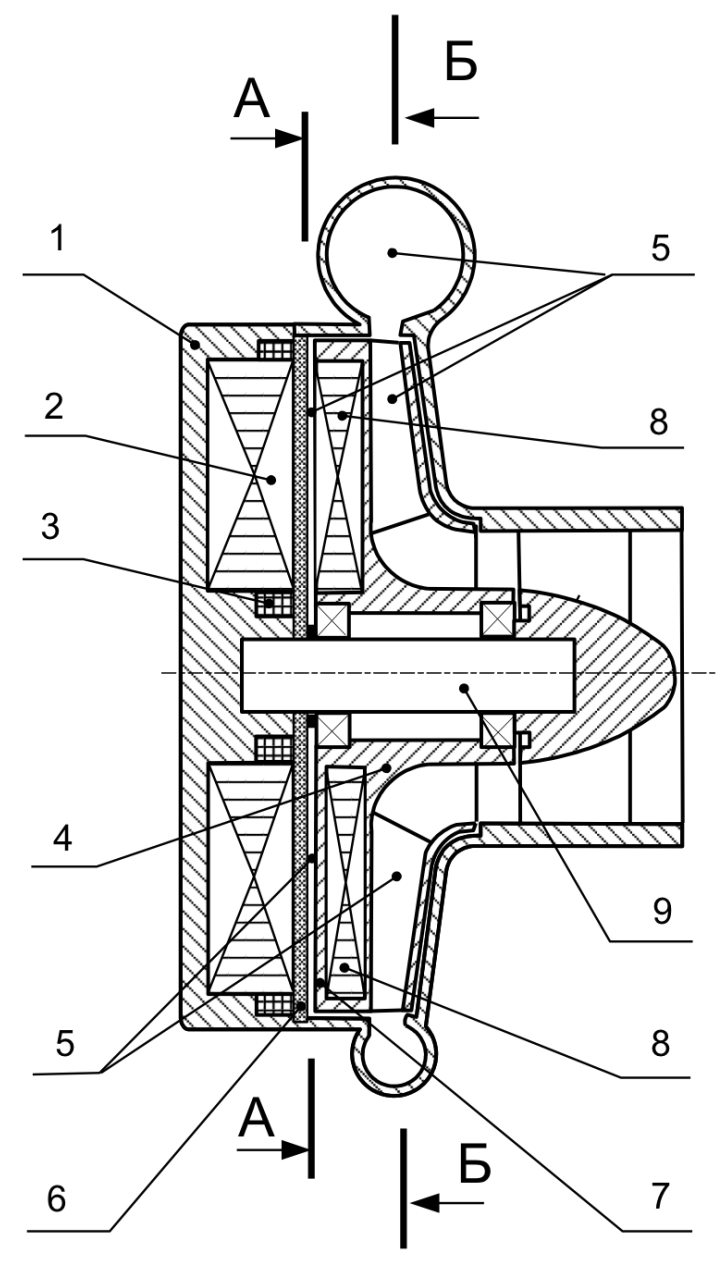

Fig. 5. Axial centrifugal motor-pump

Operational experience has shown that the disadvantage of such axial centrifugal pump motor is the presence of a large air gap between the stator and rotor cores, leading to an increase in the magnetic resistance, and therefore to an increase in the currents required to produce the magnetic flux (magnetization current), so there is an increase in the required section of the wires of the windings and, accordingly, to the deterioration of the mass-dimensions of the axial centrifugal motor-pump, its cost, energy loss and efficiency. A significant disadvantage of such axial centrifugal pump motor, as well as of any axial electric machine, is also the presence of a large axial electromagnetic force caused by the attraction of the rotor and stator. This effort leads to the premature failure of the bearing assemblies, which reduces the reliability of the pump motor. And the presence of bearing assemblies, necessary in the prototype, allows the rotation of the rotor, complicates the design of the pump motor as a whole. In addition, the skewing of the rotor relative to the stator, caused by a large axial electromagnetic force, can lead to a jamming of the rotor, which reduces the reliability of the pump unit as a whole.

To reduce the gap between the stator and the rotor of the pump motor, to improve its mass-dimensions, to reduce the cost and energy losses, to increase the efficiency, to simplify the design and to increase the reliability of the pump unit, an axial-motor pump [10] was developed (Fig. 6).

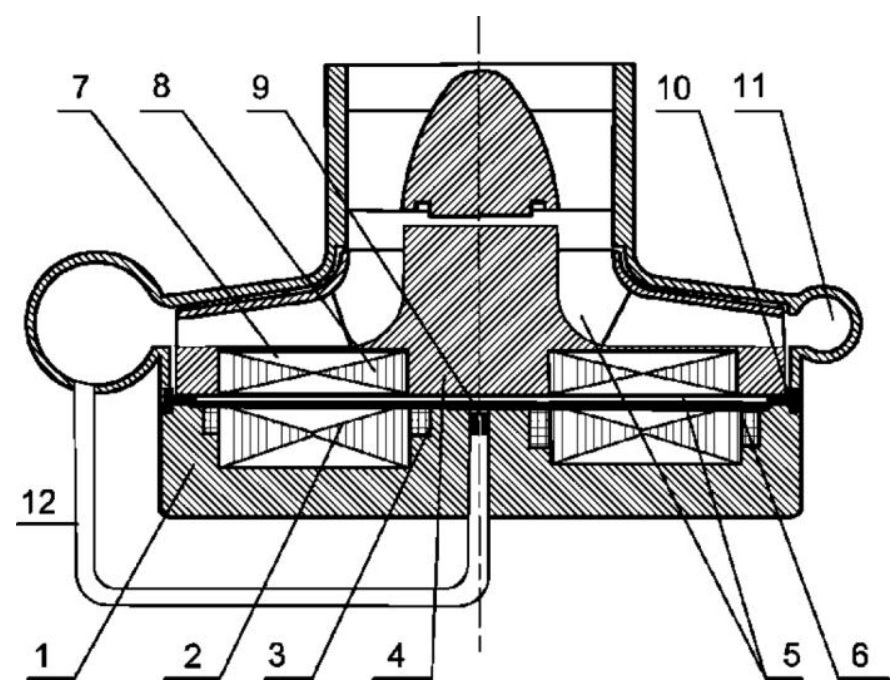

Fig. 6. Axial motor-pump

The axial motor-pump comprises a body 1, a stator 2 of an electric motor assembled therein, which is an axial laminated magnetic circuit with grooves, which includes three-phase coil 3 and impeller-rotor 4. Stator 2 of the electric motor is hermetically sealed from flowing part 5 of the pump by thin membrane 6 of dielectric material and has calibrated hole 9 in the center, and impeller-rotor 4 made of a light aluminum alloy has laminated axial magnetic circuit 8 , which includes short-circuited winding 7 . Between rotor 4 and the stator 2, there is T-ring 10, and output channel 11 of the pump motor is formed with bypass pipe 12 connected to calibrated hole 9 .

Axial motor-pump pumps the fluid according to the wellknown principle of operation of conventional centrifugal pumps, described above. The adjustment of the gap between the rotor and the stator is as follows. When the pressure in the cavity between stator 2 and rotor 4 increases, rotor 4 tends to move away from stator 2 in the axial direction due to the 
known laws of hydraulics. The amount of displacement determines the width of the gap between rotor 4 and ring 10 . The width of the gap, in turn, determines the amount of pressure in the cavity of the rotor-stator ring. As the gap increases, the amount of drained fluid increases, and the pressure in the rotor-stator-ring cavity decreases. Thus, the gap between the rotor and stator of the motor-pump is automatically adjusted. If there is a distortion of the rotor 4 in the sector in which the removal of rotor 4 from stator 2 is the greatest, the width of the slit increases, thereby reducing the pressure under rotor 4 in this sector. In the opposite sector, the width of the gap decreases, and the pressure, respectively, increases. Inequality of the pressure forces in the opposite sectors of rotor 4 leads to the appearance of forces and moments that eliminate the resulting skew, that is, automatically adjusts the gap between stator 2 and rotor 4 .

The developed engine-pump unlike the previous design and other known centrifugal pumps allows one to reduce a gap between a stator and a rotor of the engine-pump, having limited only height of ring of the T-shaped section; to improve the weight and dimensions of the pump unit, to reduce its cost. In addition it helps to simplify the design and to increase the reliability of the pump unit as a whole, by performing a motor pump without bearings and shaft (axis). It will also enables one to reduce energy losses and, accordingly, to improve efficiency by reducing the air gap between the stator and the rotor, which leads to a reduction of the loss of magnetic flux scattering.

Another possible solution of the problem is reducing the gap between the stator and the rotor of the pump motor, improving its weight and size parameters, reducing the cost and energy losses, improving efficiency, simplifying the design and increasing the reliability of the pump unit. Along the perimeter of the stator of the motor-pump, annular grooves of a semicircular cross-section are made, into which dielectric rolling bodies are laid, and along the perimeter of the impellerrotor of the motor-pump, annular grooves of a semicircular section are also made, through which the impeller-rotor of the motor-pump is mounted on dielectric rolling elements with the possibility of rotation.

Fig. 7 shows the general view of the axial centrifugal motor-pump in the section, and the enlarged image of the annular grooves of the semicircular section, in Fig. 8 there are sections $\mathrm{AA}$ and $\mathrm{BB}$

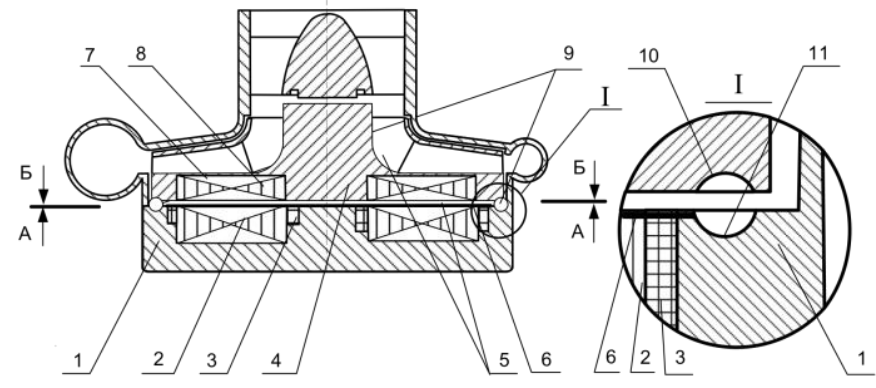

Fig. 7. General view of axial centrifugal motor-pump in section

The axial centrifugal motor-pump comprises a body 1 , a stator 2 of an electric motor assembled therein, which is an axially laminated magnetic circuit with grooves in which three-phase winding 3 is laid, and impeller-rotor 4 around which circumferential grooves 10 of a semicircular crosssection are formed (Figure 7). Stator 2 of the electric motor is hermetically sealed from flowing part 5 of the pump by a thin diaphragm 6 of dielectric material, and impeller-rotor 4 made of a light aluminum alloy has laminated axial magnetic circuit 8 into which short-circuited winding 7 is arranged. Impellerrotor 4 is mounted due to dielectric rolling elements 9 by way of annular grooves 9, arranged in annular grooves 11 of a semicircular cross-section (Figure 7) along the perimeter of stator 2.

\section{$\underline{A-A}$}

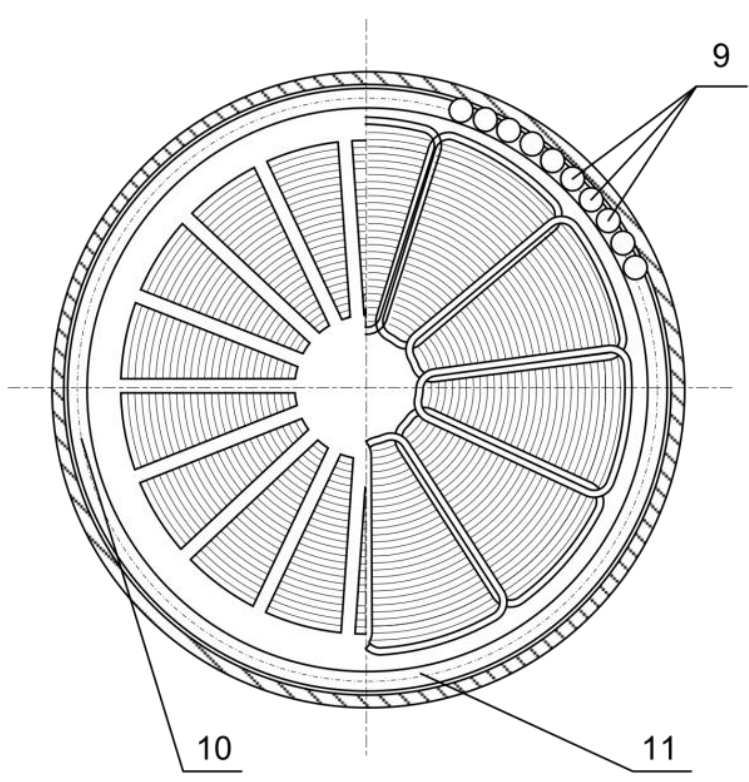

Fig. 8. Enlarged image of annular grooves of semicircular section

\section{CONCLUSION.}

Thus, the proposed design solutions, in contrast to the known ones, allow one: to reduce the gap between the stator and the rotor of the motor-pump by increasing the rigidity of the structure due to the large support surface of the impellerrotor because of the location of the rolling elements along the whole perimeter of the stator and impeller, reducing the size of the gap only by the state of the surfaces (roughness and flatness) of the stator and the rotor. Besides, they can improve the mass-dimensions of the pump set, reduce its cost, simplify the design and to increase the reliability of the pump unit as a whole by performing a motor-pump without bearings and shaft (axis). They will also make it possible to reduce energy losses and, accordingly, to improve efficiency by performing rolling bodies from dielectric, thereby reducing the heating of the pumped liquid by reducing the losses due to eddy currents and magnetic hysteresis in the rolling elements. 


\section{References}

[1] G.M. Georgievskij, M.G. Georgievskij, S.M. Smirnov, A.E. Sherstnev, N.A. Shashin, Patent for invention RUS No. 2275532. Oil product transfer pump, 10.08.2005. It is published on 27.04.2006. Bul. No. 12.

[2] V.N. Ivankovsky, Oil and gas field equipment. Moscow: CentrLitNefteGas, 2006, p. 35-38.

[3] S.G. Valjukhov, A.I. Zhitenev, S.V. Zhitenev, S.A. Dedov, S.V. Pechkurov, M.V. Feropontov, N.P. Selivanov, Patent for invention RUS No. 2449173. Centrifugal pump. 26.05.2011. It is published on 27.04.2012. Bul. No. 12.

[4] A.V. Trulev, I.N.Lozhkina, Patent for invention RUS No. 2586801. Submersible multi-phase pump blade. 29.06.2015. It is published on 10.06.2016. Bul. No. 16.
[5] D.M. Bolshakov, A.T. Nagiev, V.V. Zherebtsov, Patent for invention RUS No. 2625420. Submersible displacement pump, 02.11.2015. It is published on 10.05.2017. Bul. No. 13.

[6] J.M. Merkushev, A.V. Kraev. O.N. Vinogradov, Patent for invention RUS No. 2353814. Dispersing stage of submersible multistage centrifugal pump, 10.07.2007. It is published on 27.04.2009. Bul. No. 12.

[7] D.F. Gimkaev, Patent for invention RUS No. 2531487. Stage of centrifugal well pump, 03.07.2013. It is published on 20.10.2014. Bul. No. 29.

[8] B.A. Joffe, I.M. Kirk, Inventor's certificate USSR No. 212066. Hermetic centrifugal pump. It is published on 30.06.1978.

[9] B.Kh. Gaitov, Ya.M. Kashin, M.I. Ryabukhin, T.B. Gaitova, Patent for invention RUS No. 2284426. Axial centrifugal motor-pump. 20.04.2005. It is published on 27.09.2006. Bul. No. 27.

[10] B.Kh. Gaitov, Ya.M. Kashin, M.I. Ryabukhin, T.B. Gaytova, A.Ya. Kashin, Patent for invention RUS No. 2340974. Axial motor-pump, 26.10.2007. It is published on 10.12.2008. Bul. No. 34 